Repository of the Max Delbrück Center for Molecular Medicine (MDC) Berlin (Germany)

http://edoc.mdc-berlin.de/8815/

\title{
Mutations in MYOZ1 as well as MYOZ2 encoding the calsarcins are not associated with idiopathic and familial dilated cardiomyopathy
}

Maximilian G. Posch, Andreas Perrot, Rainer Dietz, Cemil Özcelik, Sabine Pankuweit, Volker Ruppert, Anette Richter, and Bernhard Maisch 


\title{
Mutations in MYOZ1 as well as MYOZ2 encoding the calsarcins are not associated with idiopathic and familial dilated cardiomyopathy
}

\author{
Maximilian G. Posch, Andreas Perrot, Rainer Dietz, Cemil Özcelik, Sabine Pankuweit, \\ Volker Ruppert, Anette Richter, and Bernhard Maisch \\ ${ }^{1}$ Charité - Universitätsmedizin Berlin/Cardiology at Campus Buch \& Virchow Klinikum, Helios-Kliniken and Max- \\ Delbrück Center for Molecular Medicine, Wiltbergstrasse 50, 13125 Berlin, Germany \\ ${ }^{2}$ Department of Internal Medicine and Cardiology, Philipps University Marburg, Baldingerstrasse, 35043 Marburg, \\ Germany
}

\section{Dear Editor,}

We have read the article of Arola and colleagues [1] with great interest. The authors report a candidate gene approach in the MYOZ1 gene encoding calsarcin-2 in 185 patients with idiopathic dilated cardiomyopathy (iDCM). No disease associated mutation was identified by use of denaturing high-performance liquid chromatography (DHPLC).

We have examined a cohort of 61 patients with proven familial dilated cardiomyopathy (fDCM). fDCM was diagnosed when two or more affected relatives were present in one family (range: $2-7$; mean: 3.2). Clinical examination was performed according to the European guidelines for investigation of familial dilated cardiomyopathy [2] as described previously [3]. We directly sequenced all coding exons (2-6) with flanking intronic portions of MYOZ1 by use of $A B I$ Big Dye chemistry as described in detail in [4]. In contrast to Arola et al. [1], we additionally analyzed exonic fragments (2-6) of MYOZ2 encoding the cardiac calsarcin-1.

We also identified the L255L (CTG > CTT) variant as described in [1]. Five fDCM patients were heterozygous L255L carriers. However, no other genetic variant in MYOZ1 could be detected by us. Sequencing of MYOZ2 resulted in identification of two synonymous polymorphisms (A79A and E153E). These heterozygous variants were uniquely detected in two individuals with fDCM. All identified polymorphisms in MYOZ1 and MYOZ2 are represented in Fig. 1.

Although DHPLC has been proven to be a sensitive method for mutational analysis, one can not completely exclude that some variants might have escaped the analysis of Arola and colleagues. However, direct sequencing constitutes the gold standard of genetic analysis almost excluding false negative results in candidate gene approaches. Furthermore, screening in patients with a hereditary form of DCM offers several advantages: first, the prevalence of pathogenic mutations should be higher in patients with familial DCM. Further, identified unknown variants can easily be checked for cosegregation with the disease in the family pedigrees. Secondly, in case of negative results (as described here) the probability that the respective candidate gene does not constitute a relevant disease gene is higher when screening a cohort with familial disease.

The most important point to address to the work described by Arola et al. [1] is that the analysis should not be restricted to MYOZ1 when investigating the genetic etiology of DCM. Indeed, MYOZ1 is abundantly expressed in skeletal muscle, but cardiomyocytes reveal a very weak mRNA expression of MYOZ1 [5]. Conversely, MYOZ2 encoding calsarcin-1 is predominantly expressed in cardiomyocytes and to lesser extends in skeletal muscle $[5,6]$. Calsarcin-1 is located at the sarcomeric Zdisk and interacts with DCM disease genes like telethonin and a-actinin. Furthermore, conditional MYOZ2 knock out mice reveal heart failure and extensive dilation of both ventricles after aortic banding [7]. Therefore, MYOZ2 represents a more plausible candidate gene for DCM.

However, taking together the results from Arola et al. [1] with results from a recent candidate gene approach analyzing MYOZ2 [8], pathogenic mutations in MYOZ1 and MYOZ2 could not be identified in 185 and 19 patients with idiopathic DCM patients, respectively. Additionally, we have excluded mutations in both genes in a cohort of 61 carefully selected families with fDCM using a highly sensitive screening method.

In conclusion, one can state that mutations in MYOZ1 and MYOZ2 are at least very rare events as an underlying disease mechanism for idiopathic or familial DCM.

We acknowledge the support of the Berlin Institute for Heart Research (BIHR-VH-VI 152) funded by the Helmholtz Gemeinschaft, research grant from CharitéUniversitätsmedizin Berlin and the German Heart Failure Network (GHFN).

\section{Corresponding Author}

Maximilian G. Posch, Charité - Universitätsmedizin Berlin/Cardiology at Campus Buch \& Virchow Klinikum, Helios-Kliniken and Max-Delbrück Center for Molecular Medicine, Wiltbergstrasse 50, 13125 Berlin, Germany. E-mail address: maximilian.posch@charite.de

\section{Referenzen}

1. A. Perrot, H.H. Sigusch, H. Nagele, J. Genschel, H.B. Lehmkuhl, R. Hetzer, C. Geier, V. Leon Perez, D. Reinhard, R. Dietz, K.J. Osterziel, H.H. Schmidt, Genetic and phenotypic analysis of dilated cardiomyopathy with conduction system disease: demand for strategies in the management of presymptomatic lamin A/C mutant carriers, Eur. J. Heart Fail. 8 (2006) 484-493.

2. C. Geier, A. Perrot, C. Ozcelik, P. Binner, D. Counsell, K Hoffmann, B. Pilz, Y. Martiniak, K. Gehmlich, P.F. van der Ven, D.O. Furst, A. Vornwald, E. von Hodenberg, P. Nurnberg, T. Scheffold, R. Dietz, K.J. Osterziel, Mutations in the human muscle LIM protein gene in families with hypertrophic cardiomyopathy, Circulation 107 (2003) 1390-1395. 
3. G. Faulkner, A. Pallavicini, A. Comelli, M. Salamon, G. Bortoletto, C. levolella, S. Trevisan, S. Kojic, F. Dalla Vecchia, P. Laveder, G. Valle, G. Lanfranchi, FATZ, a filamin-, actinin-, and telethonin-binding protein of the Z-disc of skeletal muscle, J. Biol. Chem. 275 (2000) 41234-41242.

4. N. Frey, J.A. Richardson, E.N. Olson, Calsarcins, a novel family of sarcomeric calcineurin-binding proteins, Proc. Natl. Acad. Sci. USA 97 (2000) 14632-14637.

5. N. Frey, T. Barrientos, J.M. Shelton, D. Frank, H. Rutten, D. Gehring, C. Kuhn, M. Lutz, B. Rothermel, R. Bassel-Duby, J.A. Richardson, H.A. Katus, J.A. Hill, E.N. Olson, Mice lacking calsarcin-1 are sensitized to calcineurin signaling and show accelerated cardiomyopathy in response to pathological biomechanical stress, Nat. Med. 12 (2004) 1336-1343.

6. R. Zeller, B.T. Ivandic, P. Ehlermann, O. Mucke, C. Zugck, A. Remppis, E. Giannitsis, H.A. Katus, D. Weichenhan, Large-scale mutation screening in patients with dilated or hypertrophic cardiomyopathy: a pilot study using DGGE, J. Mol. Med. 84 (2006) 682-691.

a MYOZ1 (Calsarcin-2)

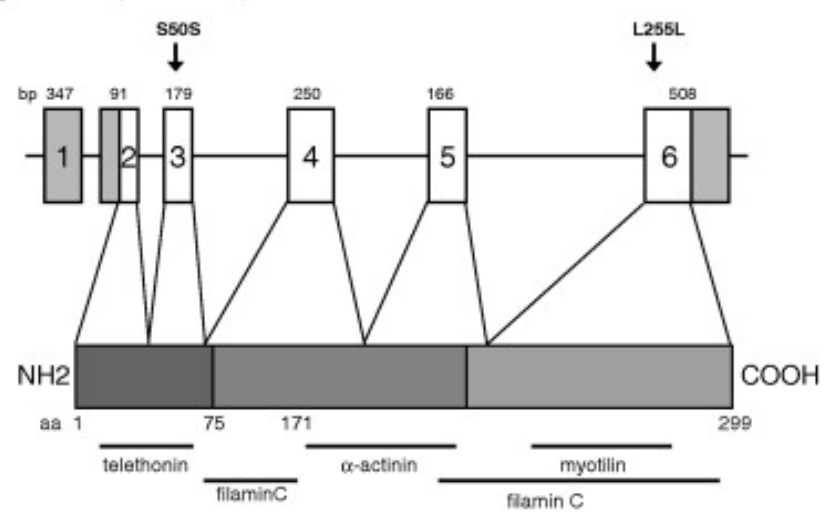

b MYOZ2 (Calsarcin-1)

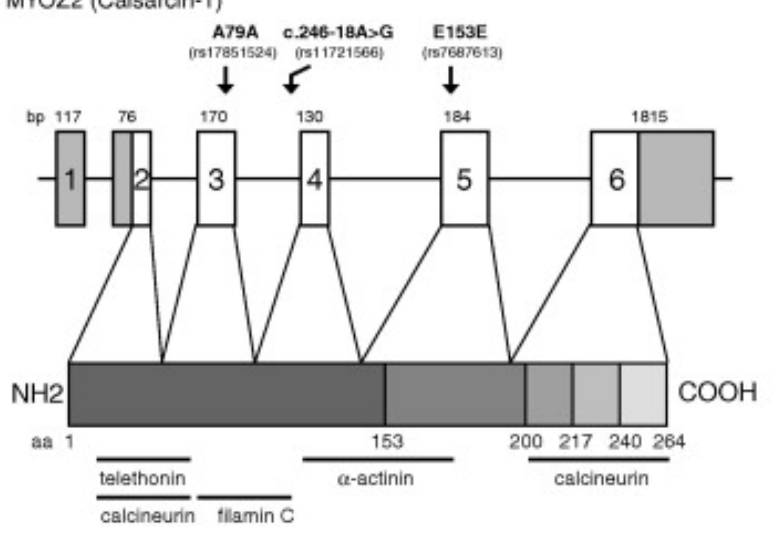

Fig.1: Schematic representation of the gene structure of MYOZ1 (NM_021245) encoding calsarcin-2 and MYOZ2 (NM_016599) encoding calsarcin-1 with the variants identified by Arola et al. [1] and us indicated. Open boxes represent exons with the number of coding nucleotides above and UTRs shaded. The S50S variant in MYOZ1 was described by Arola et al. [1], whereas the L255L variant in MYOZ1 was identified by Arola et al. and us (allelic frequency $8.2 \%$ ). The A79A and E153E polymorphisms in MYOZ2 were identified in two patients of our fDCM cohort, respectively. Many different and overlapping protein-protein interaction domains have been described for calsarcin-1 and calsarcin-2 in different studies [5] and [6]. 